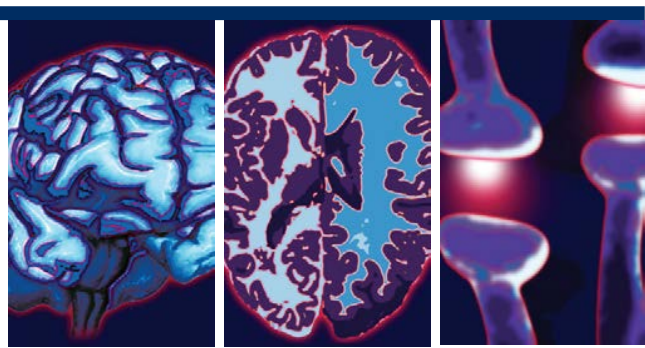

\title{
The Mediating Effect of Depression in Religiosity and Cognitive Function among Chinese Muslim Elderly
}

\author{
Yan Sun'1, Wanrui Ma², Yanxiao Wu'1, Harold G. Koenig ${ }^{1,3,4}$, Zhizhong Wang ${ }^{1,+}$
}

\begin{abstract}
The current study examined the association between religiosity and mild cognitive impairment $(\mathrm{MCl})$, along with the mediating role of depression, in Chinese Muslim elderly sample. 1,347 community Muslims aged 55 years or older were recruited in Ningxia, China. The MiniMental State Exam, the Geriatric Depression Scale, and the Duke University Religion Index were administered. Structural equation modeling (SEM) was used to detect the mediation effect. Approximately $21.3 \%$ participants reported experiencing mild or severe depressive symptoms and $25.7 \%$ met criteria for $\mathrm{MCl}$. Religiosity was associated with a lower risk of $\mathrm{MCl}(\mathrm{OR}=0.83, \mathrm{P}<0.05)$, while depression was associated with a greater risk of $\mathrm{MCl}(\mathrm{OR}=1.70$, $\mathrm{P}<0.01)$. SEM analysis revealed that depression significantly mediated the association between the religiosity and $\mathrm{MCl}$ with an effect ratio of 0.33 (explaining $33.0 \%$ of the total variance). In summary, religiosity was related to cognitive functioning partly due to its inverse relationship with depression in older Chinese Muslims.
\end{abstract}

\section{Keywords}

Depressive, Mild cognitive impairment, Mediating effect, Religiosity, Muslim

\section{Introduction}

It is estimated that by 2050 the number of population over the age of 60 will be 2 billion globally [1]. A negative consequence of the rapidly aging of world population is the increased prevalence of cognitive disorders; include dementia and mild cognitive impairment (MCI) [2]. The determinants of cognitive impairment include age, education, smoking, physical activity, and social engagement [3-5]. Besides the above factors, systematic reviews have shown that depression is associated with an increased risk of developing dementia [6] and cognitive decline [7]. Moreover, depression as a risk factor has been found interacted with genetic factors
\end{abstract}

to increase risk of cognitive decline. Individuals with both depressive symptoms and one or more copies of the apolipoprotein E (APOE) 4 allele experience more cognitive decline than depressed persons without copies of the APOE 4 allele, leading to a greater risk of Alzheimer disease (AD) [8]. Research has also indicated that depression by itself may increase the risk of dementia $[9,10]$.

As a social determinant of health, religious involvement has been found contributed to a lower risk of cognitive decline [11-14]. A review of this research concluded that higher levels of religiosity in Alzheimer's patients were associated with a slower rate of cognitive and behavioral

\footnotetext{
'Department of Epidemiology, School of Public Health and Management, Ningxia Medical University, Yinchuan, 750004, China ${ }^{2}$ Comprehensive Department, General Hospital of Ningxia Medical University, Yinchuan, 740004, China

${ }^{3}$ Departments of Psychiatry and Medicine, Duke University Medical Center, Durham, NC 27710, USA

${ }^{4}$ Department of Medicine, King Abdulaziz University, Jeddah, 21589, Saudi Arabia

${ }^{\dagger}$ Author for correspondence: Zhizhong Wang, Department of Epidemiology, School of Public Health and Management, Ningxia Medical University, Yinchuan, 750004, China, email:wzhzh_lion@126.com
} 
decline, with a corresponding significant reduction in caregiver burden [15]. This was explained by an increase in social integration, better behavioral self-control [16], and fewer life event stresses among those who are religiously active [17]. Religious involvements have also been associated with lower depression prevalence $[18,19]$, a 14 years follow-up study in Canada demonstrated that more religious involvements reduced risk of developing major depression by $22 \%$ [20].

What then is the relationship among religiosity, depression, and MCI or cognitive decline? ReyesOrtiz et al examined how church attendance modified the effect of depressive symptoms on cognitive function in Mexican Americans. Among participants with high depressive symptoms, those infrequent church attendances experienced a greater decline in Mini-Mental State Examination (MMSE) scores than those frequent church attendances [21]. Most recently, Foong et al revealed that the intrinsic religiosity mediated the association between depression and cognitive function after controlling for selected wide range of socio-demographic characteristics [22]. However, much more needs to be known about this relationship. First, both above studies did not indicate how much depression mediated the association between religiosity and MCI. Raykov indicated that "If an indirect effect does not receive proper attention, the relationship between two variables of interest may not be fully considered" [23]. Second, the association between religion and mental health is robust across cultures. Most studies of religiosity and cognitive function were conducted in highly religious societies and in Christian populations. To our knowledge, little research has examined the relationship between religion and cognitive function, or how depression influences this relationship, in China where religion is less practiced and encouraged.

To address those gaps in the literature, the present study sought to examine the mediating effect of depressive symptoms on the relationship between religiosity and cognitive function using structural equation model in a communitydwelling sample of Chinese Muslims.

\section{Methods}

\section{- Participants and field procedure}

Participants in present study were acquired from Ningxia Hui Autonomous Region, a northwestern province of China, which has a population of 2.2 million residents of Hui ethnicity (constituting $36.1 \%$ of the total population in the province). This ethnic group is composed almost entirely of Muslims. A cluster sampling method was used to identify participants in this study. Three communities were contacted and agreed to participate in the face-to-face survey. Inclusion criteria for the participants were (1) individuals living in the selected communities for at least six months; (2) aged 55 years or older; and (3) having a verified Hui ethnicity identification card. Excluded were those with a history of psychosis, severe hearing loss, vision loss, and language barriers. Of a total of 1,665 community residents eligible for the survey, 1,347 ( $80.9 \%$ of the total sample) agreed to participate and completed the full questionnaire were included in the final analysis.

Face-to-face surveys were conducted by trained interviewers at the time they attended a health examination at community health care centers. The health examination is part of a public health service program supported by the local government. All those aged fifty years or older are eligible to receive free health examinations once a year at their local community health care centers.

\section{- Ethical considerations}

The study was conducted in accordance with the Helsinki Declaration, World Medical Association and approved by the Institutional Review Board at Ningxia Medical University (document number: 2015151). Written informed consent was obtained from all participants.

\section{- Measurement}

Sociodemographic information was collected including age, gender, marital status, living status (living alone or not), educational level, and occupation. The Activities of Daily Living Scale (ADL) was used to measure the physical function.

\section{- Outcome variables}

Cognitive function was measured using the Chinese version of the MMSE [24]. The MMSE is short, easy to administer, and has good reliability and validity as a screening tool for cognitive impairment [25]. The MMSE consists of 19 questions and has a score ranged from 0 to 30. It assesses five different domains of cognitive function: (1) orientation with a maximum of 10 points, (2) memory with a maximum of 6 points, 
(3) attention and calculation with a maximum of 5 points, (4) language with a maximum of 8 points, and (5) visuospatial constructional ability (copying intersecting pentagons) with the maximum of 1 point. The determination of the normal score is closely related to the level of education of the subjects [26]. MCI was diagnosed according to Peterson's criteria [27] with modification as Cui et al suggested: MMSE was used assessing normal cognitive function instead of verbal memory test, and the educational level was taken into account when determining cutoff as: For those who were illiterate with MMSE $\leq 17$; for those with only a primary school education, the threshold was MMSE $\leq 20$; for high school graduates or above, the threshold was MMSE $\leq 24$ [28]. In addition, participants were asked the question "Have you had a significant decline in your memory recently?"

\section{- Independent variables}

Religiosity was measured using the Chinese version of the 5-item Duke University Religion Index (DUREL), a comprehensive and brief measure of organizational religious activity (OR), non-organizational religious activity (NOR), and intrinsic religiosity (IR). The DUREL has a total score range from 5 to 27, higher scores indicate higher level of religiosity [29]. The Chinese version of the DUREL is a reliable and valid measure of religious involvement that can be used to assess the relationship with physical and psychological well-being in Chinese respondents [30]. In the present study, high religiosity was defined as praying more than once a day and attending religious services at least once per week, and indicating that religion was important or very important in their life (i.e., corresponding to a DUREL total score $>24$ ) [31].

The Geriatric Depression Scale (GDS) was used to assess depressive symptoms. The GDS is a frequently used and well-validated screening measure for depression among older adults [32]. It consists of 30 self-report items that assess a broad range of depressive symptoms using a simple yes/ no response format. GDS scores ranged from 0 to 30 , with higher score indicating more severe depressive symptom [33]. Regarding total score cutoffs, 0 to 9 indicates normal mood; 10 to 19 indicates mild depressive symptoms; and 20 to 30 indicates severe depressive symptoms [34]. The Chinese version of the GDS has been shown to be a reliable and valid measure of depression in rural and urban populations [35].

\section{- Statistical methods}

The differences in socio-demographic variables between participants with MCI and those with normal cognition were tested using the chi-square test for categorical variables, and the Student's $t$ test for age, the Wilcoxon rank-sum test for DUREL. Logistic regression models were used to examine the association among religiosity, depression and MCI. All analysis was performed using the SPSS 22.0 (IBM Corporation, Armonk, NY, USA), and significance level was set at $\mathrm{p}<0.05$, with two-tailed test.

Structural equation modeling (SEM) coupled with the bias-corrected bootstrap method (2000 replicates) was employed to examine the mediating effect of depression on the relationship between religiosity and cognition function [36,37]. Post-hoc modifications were performed with reference to the modification index. A mediating effect model was used since the bootstrapping confidence intervals did not include zero [38]. Both the direct effect and mediating effect were estimated using AMOS version 21.0 (IBM Corporation, Armonk, NY, USA). Several indices of model fit were calculated, including the root mean square error of approximation (RMSEA), where less than 0.08 is acceptable; Goodness of Fit Index (GFI), where values larger than 0.90 indicate a good model fit; the Values of Comparative Fit Index (CFI) and the Tucker Lewis Index (TLI), where values of 0.90 or higher indicate acceptable fit.

\section{Results}

\section{- Demographic characteristics of participants}

As displayed in Table 1, the average age of participants was $65.1 \quad(\mathrm{SD}=6.0)$ years, with a range from 55 to 84 years. Nearly half of participants was female $(51.9 \%)$, and were illiterate $(57.2 \%)$. Only $3.9 \%$ of participants lived alone, and most participants $(96.2 \%)$ were married. $21.3 \%$ participants reported mild or severe depressive symptoms; concerning cognitive function, $25.7 \%$ met criteria for MCI. Participants with MCI were older than those without cognitive impairment, were more likely to be female, were more likely having depressive symptoms, and were less likely to score high on religiosity.

\section{- Categorical analyses}

As Table 2 shows, categorical analyses revealed that religiosity was inversely related to risk of 


\section{Research Zhizhong Wang}

\begin{tabular}{|c|c|c|c|c|c|}
\hline Variables & $\begin{array}{l}\text { Total } \\
\mathrm{n}=1,347\end{array}$ & $\begin{array}{l}\mathrm{MCl} \\
\mathrm{n}=346\end{array}$ & $\begin{array}{l}\text { Normal } \\
n=1,001\end{array}$ & t/Chi & $P$ value \\
\hline Age, mean (SD), years & $65.1(6.0)$ & $65.2(5.7)$ & $64.2(5.3)$ & 6.84 & $<0.001$ \\
\hline DUREL, mean (SD) & $24.8(3.5)$ & $24.2(4.1)$ & $25.0(3.2)$ & 3.57 & $<0.001$ \\
\hline Gender, female, n (\%) & $699(51.9)$ & $210(60.7)$ & 489 (48.9) & 14.45 & $<0.001$ \\
\hline Urban/rural, rural, n (\%) & $1,126(83.7)$ & $292(25.9)$ & 834 (74.1) & 0.22 & 0.641 \\
\hline Education & & & & 31.62 & $<0.001$ \\
\hline Illiterates, n (\%) & $770(57.2)$ & $163(47.1)$ & $607(60.6)$ & & \\
\hline Primary school, n (\%) & $338(25.1)$ & $89(25.7)$ & $249(24.9)$ & & \\
\hline > Junior high school, n (\%) & $239(17.7)$ & $94(27.2)$ & $145(14.5)$ & & \\
\hline Living alone, yes, n (\%) & $52(3.9)$ & $17(4.9)$ & $35(3.5)$ & 1.39 & 0.238 \\
\hline Marriage time, $>=2, \mathrm{n}(\%)$ & $51(3.8)$ & $14(4.0)$ & $37(3.7)$ & 0.09 & 0.769 \\
\hline Depressive symptoms & & & & 7.14 & 0.028 \\
\hline Normal mood, $\mathrm{n}(\%)$ & $1,060(78.7)$ & $255(73.7)$ & $805(80.4)$ & & \\
\hline Mild depressive, n (\%) & $216(16.0)$ & 67 (19.4) & 149 (14.9) & & \\
\hline Severe depressive, n (\%) & $71(5.3)$ & $24(6.9)$ & $47(4.7)$ & & \\
\hline High Religiosity, yes, n (\%) & $1,042(77.4)$ & $246(71.1)$ & 796 (79.5) & 10.41 & 0.001 \\
\hline Hypertension, yes, n (\%) & $429(31.8)$ & $112(32.4)$ & $317(31.7)$ & 0.05 & 0.809 \\
\hline
\end{tabular}

\begin{tabular}{|c|c|c|c|c|c|c|}
\hline \multirow{2}{*}{ Variables } & \multicolumn{2}{|l|}{ Model 1} & \multicolumn{2}{|l|}{ Model 2} & \multicolumn{2}{|l|}{ Model 3} \\
\hline & $P$ value & OR $(95 \% C l)$ & $P$ value & $O R(95 \% C l)$ & $P$ value & OR $(95 \% C l)$ \\
\hline Religiosity (high) & 0.001 & $0.63(0.48,0.83)$ & 0.014 & $0.69(0.52,0.93)$ & 0.330 & $0.83(0.57,1.20)$ \\
\hline Depressive (yes) & 0.009 & $1.46(1.10,1.95)$ & 0.020 & $1.42(1.05,1.92)$ & 0.039 & $1.70(1.02,2.80)$ \\
\hline Age ( $>=65$ Year) & NA & NA & $<0.001$ & $1.58(1.22,2.04)$ & 0.001 & $1.55(1.20,2.01)$ \\
\hline Education (illiterate) & NA & NA & $<0.001$ & $0.39(0.30,0.52)$ & $<0.001$ & $0.39(0.30,0.52)$ \\
\hline gender (female) & NA & NA & $<0.001$ & $2.29(1.73,3.03)$ & $<0.001$ & $2.23(1.68,2.96)$ \\
\hline Depressive $\times$ Religiosity & NA & NA & NA & NA & 0.244 & $0.68(0.36,1.29)$ \\
\hline
\end{tabular}

MCI. In contrast, depression was positively associated with MCI (model 1). After controlling for socio-demographic variables, these findings persisted significant (model 2). The association between religiosity and MCI, however, disappeared when depression was controlled for in the model (model 3), indicating that depression fully mediated the relationship between religiosity and MCI.

\section{- Analyses of continuous variables}

The results of bivariate correlations when independent variables were left as continuous are presented in Table 3. After controlling for sociodemographic variables (age, gender, education), the DUREL score was positively correlated with MMSE score $(\mathrm{P}<0.001)$; the DUREL was negatively correlated with GDS score $(\mathrm{P}<0.001)$; and the GDS score was negatively correlated with MMSE score $(\mathrm{P}<0.001)$.

\section{- SEM results for mediation effects}

A mediation model was developed as shown in
Figure 1. After the post-hoc modifications were performed, the final model yielded good fit of the data $(\mathrm{x} 2=5.47$, RMSEA $=0.058, \mathrm{GFI}=$ $0.971, \mathrm{CFI}=0.943$, TLI $=0.921, \mathrm{IFI}=0.943$, $\mathrm{NFI}=0.931)$. The total effect, direct effect and mediating effect of religiosity on cognitive function with standard errors and confidence intervals were obtained using the Bootstrap method. The results showed that the direct effect and the total mediating effect were significant $(\mathrm{P}<0.05)$. The mediation effect of depression in the relationship between religiosity and cognitive functioning accounted for $33.03 \%(0.179 / 0.542)$ of the total effect (Table 4). The PRODCLIN program test results showed that the 95\% confidence interval for this mediation $(0.117,0.263)$ and the bias-corrected program test results indicate that the $95 \%$ confidence interval was $(0.116,0.260)$, all ranges do not include 0 , indicating that the mediation paths was statistically significant. 


\begin{tabular}{|c|c|c|c|c|c|}
\hline & $M$ & SD & MMSE & GDS & DUREL \\
\hline MMSE & 22.7 & 4.5 & 1 & & \\
\hline GDS & 5.8 & 6.1 & $-0.274^{* * *}$ & 1 & \\
\hline DUREL & 24.8 & 3.5 & $0.099 * * *$ & $-0.290^{* * *}$ & 1 \\
\hline
\end{tabular}

\begin{tabular}{|c|c|c|c|c|c|c|c|}
\hline \multirow{3}{*}{ Effect } & \multirow{3}{*}{ Point estimation } & \multirow{2}{*}{\multicolumn{2}{|c|}{ Product of coefficients }} & \multicolumn{4}{|c|}{ Bootstrapping } \\
\hline & & & & \multicolumn{2}{|c|}{ Bias-corrected } & \multicolumn{2}{|c|}{ Percentile $95 \% \mathrm{Cl}$} \\
\hline & & SE & Z & Lower & Upper & Lower & Upper \\
\hline Total effect & 0.542 & 0.117 & 4.63 & 0.346 & 0.825 & 0.343 & 0.818 \\
\hline Mediating effect & 0.179 & 0.038 & 4.71 & 0.116 & 0.260 & 0.117 & 0.263 \\
\hline Direct effect & 0.363 & 0.101 & 3.59 & 0.194 & 0.594 & 0.188 & 0.589 \\
\hline \multicolumn{8}{|c|}{$\mathrm{SE}=$ Standard error $; 95 \% \mathrm{Cl}=95 \%$ confidence interval } \\
\hline
\end{tabular}

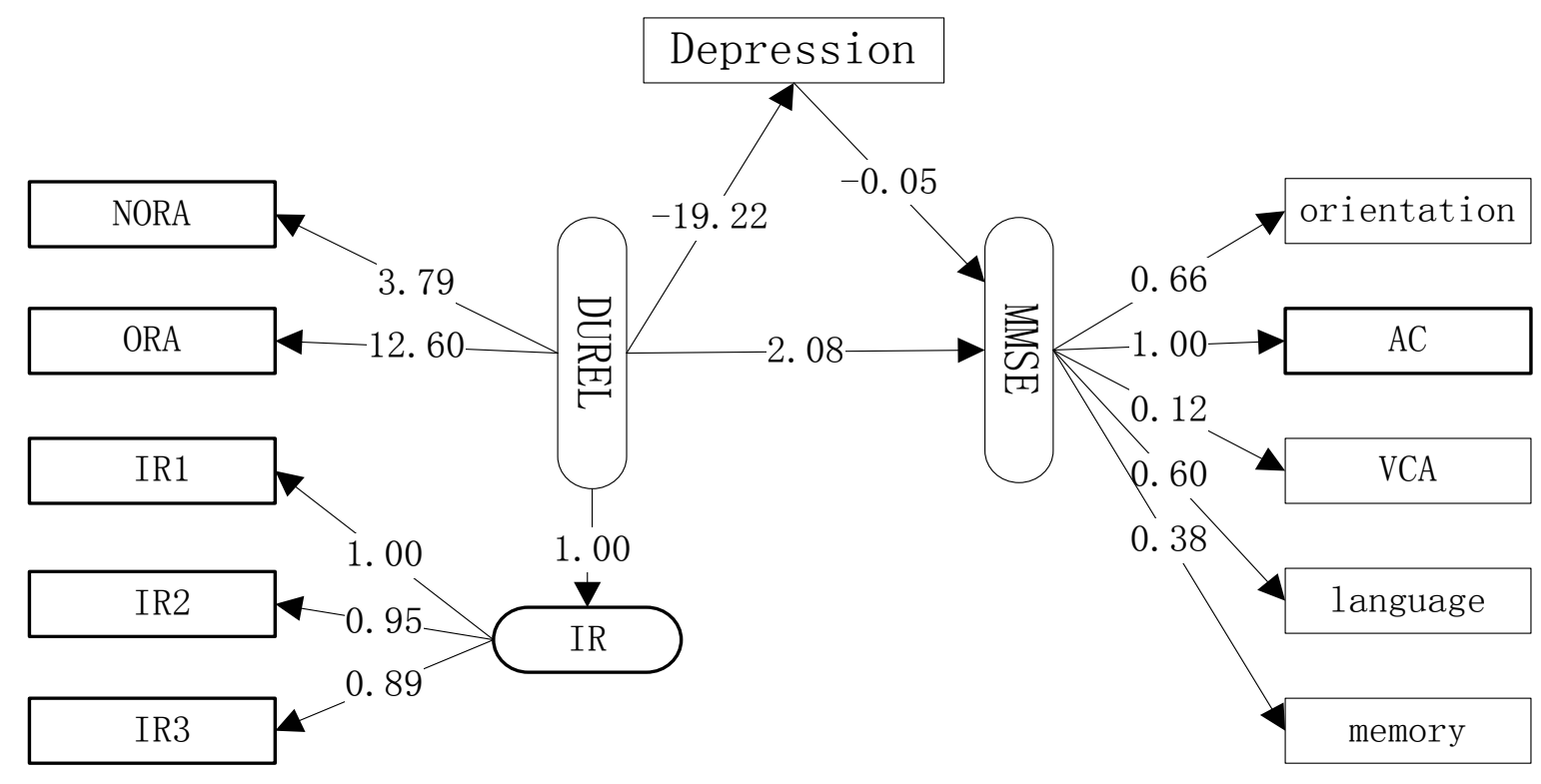

Figure 1: The mediation model of depression in the relationship between religiosity and cognitive function. ORA: Organizational religious activity, NORA: non-organizational religious activity, IR: intrinsic beliefs Religiosity, AC: Attention and calculation, VCA: visuospatial constructional ability; MMSE: Mini-Mental Stale Examination; DUREL:Duke University Religion Index; the data in the figure are normalized path coefficients.

\section{Discussion}

As hypothesized, this study found that high religiosity was associated with a reduced risk of MCI, and with the beneficial effects mediated by depression. The mediation effect accounted for $33.03 \%$ of the total effect. These findings suggest that religious involvement may be important for maintaining cognitive function over time for Chinese Muslims, which is likely because religion helps them to cope with problems as they get older. These findings are important for understanding how and why religiosity is related to cognitive function.

Compared with those who were less religious, the average depressive symptoms score was significantly higher in those who were more religious. This finding is consistent with a community-based 30-year follow-up longitudinal study in China [18] and a 14 years follow-up study in Canada, which demonstrated that religious involvement reduced the risk of developing major depression by $22 \%$ [20]. Furthermore, Paine found that religious involvement predicted lower levels of depressive symptoms in a sample of graduate students in the helping of professions $[39,40]$. Stimulating activities have been shown to help build cognitive reserve that delays the manifestation of cognitive difficulties [41]. As noted above, religious/spiritual beliefs and activities of older Chinese Muslims may help to buffer against 
life stress and promote comfort by improving positive psychosocial and physiological coping; In addition, these may increase life purpose and meaning, improve tangible resources from others who are religious, a sense of belonging and empowerment [42]. Similarly, previous research has shown a moderating effect of church attendance on the relationship between depressive symptoms and cognitive function, where higher levels of church attendance appears to have buffered the negative impact of depressive symptoms on cognitive function [21]. Using a structural equation modeling (SEM), David et al found that physical activity influenced cognition directly and reduced depression indirectly by increasing social networks [43]. Much research has shown that depression is a risk factor for cognitive decline, and older people with depression are more likely to have dementia [4447].

MCI is regarded as a stage of cognitive impairment that is intermediate between normal ageing and dementia, where impairments have not yet affected the individual's ability to function in daily life $[48,49]$. The prevalence of MCI in the current study was found to be over $25 \%$ in these community-dwelling Muslims aged 55 years or older. This rate is higher than that in the general Chinese population (with a prevalence of $14.5 \%$ in aged 55 years and older) [50]. The difference may be explained by several factors. First, the prevalence of MCI was different depending on age, sex, education, marital status, and place of residence. Participants were selected here from a poor economic region. Nearly $83.7 \%$ came from rural areas, and $83.1 \%$ were involved in peasantlevel occupations. Those sociodemographic characteristics may have influenced the high prevalence of MCI found here [50]. Second, the current study examined the association among religiosity, depression and MCI in a nonrandom community sample. The method of participant enrollment may have caused a select bias, particularly since they were recruited from community health centers.

The findings of current study have several potential implications. Although we are not suggesting that religious activity be prescribed for the people of China, with increasing openness shown by the Chinese government to religious organizations in recent years, our findings may suggest by helping elder Muslims cope with stressors, religious involvements may help them maintaining better cognitive function. We also encourage the depressed elderly patients receive antidepressant treatment as well as faith-based treatment that have well established and showed more efficacious than conventional treatment [51], could help to prevent the loss of cognitive function by reducing depression, thus helping to prevent dementia.

\section{Limitations}

The present study has several limitations. First, the cross-sectional design of this study prevents any statements about the causal direction in the relationships found. Religious involvement could either lead to better cognitive functioning or better cognitive functioning could promote increased religious involvement. Hence, a longitudinal study or clinical trial would be necessary to determine causal relationships. Second, due to the low education level of our sample ( $57.2 \%$ of the total sample were illiterate), cognitive function may have been overestimated producing measurement bias (although education level was adjusted when examining the relationships between religiosity, cognitive impairment, and depression). Furthermore, there potential confounders like economic status and occupation were not included in the statistical models, but which could influence relationships between key variables in this study.

\section{Conclusions}

The present study examined the relationship between religiosity, depression, and cognitive function in a large sample of Chinese Muslim elders. Using regression analyses and SEM, we investigated how religious involvement may influence cognitive functioning, finding depression to be a major mediator of this relationship. Religiosity appeared to have significant mediating and direct effects on cognitive function, where religious involvement was found to be important in maintaining cognitive function in these older individuals by reducing the prevalence of depressive symptoms. These findings provided information that may help guide future research and programs designed to further understand and enhance the management of MCI, perhaps through a partnership between public health practitioners and religious organizations.

\section{Acknowledgements \\ Funding was provided Open Research Fund for Top Disciplines of Public health and Prevent}




\begin{abstract}
Medicine at Ningxia Medical University (NXYLXK2017B08). These funding bodies played no role in designing the study, collecting, analyzing or interpreting the data, writing the manuscript, or deciding to submit the paper for publication.
\end{abstract}

\section{Conflicts of Interest}

The authors have no conflicts of interest to report.

\begin{abstract}
Author Contributions
SY and WZ participated in the design of the study, SY, MW, and WY conducted the data collection. SY conducted the statistical analysis and wrote the first draft of the manuscript. MW, HK and WZ oversaw the data analysis, and reviewed the draft manuscript. All the authors have read and approved the final manuscript.
\end{abstract}

\section{References}

1. Economic UPDUNDo, Affairs S. World population ageing 2007. MJSS 33(100), 328339 (2009).

2. World Health Organization. Dementia: a public health priority. Perspect. Public. Health 5(3), 123-125 (2012).

3. Flicker L. Modifiable lifestyle risk factors for Alzheimer's disease. J. Alzheimers. Dis 20(3), 803-811 (2010)

4. Deary IJ, Corley J, Gow AJ, et al. Ageassociated cognitive decline. Br. Med. Bull 92(1), 135-52 (2009).

5. Hertzog C, Kramer AF, Wilson RS, et al. Enrichment Effects on Adult Cognitive Development Can the Functional Capacity of Older Adults Be Preserved and Enhanced? Psychol. Sci. Public Interest 9(1), 1-65 (2014).

6. Da SJ, Gonçalves-Pereira $M$, Xavier $M$, et al. Affective disorders and risk of developing dementia: systematic review. Br. J. Psychiatry 202(3), 177-186 (2013).

7. Wilson RS, Barnes LL, Leon CFMD, et al. Depressive symptoms, cognitive decline, and risk of AD in older persons. Neurol 59(3), 364370 (2002).

8. Rajan KB, Wilson RS, Skarupski KA, et al. Genebehavior interaction of depressive symptoms and the apolipoprotein $E$ \{varepsilon\} 4 allele on cognitive decline. Psychosom Med 76(2), 101-8 (2014).

9. Panza F, Frisardi V, Capurso C, et al. Late-life depression, mild cognitive impairment, and dementia: possible continuum? Am J. Geriatr Psychiatry 18(2), 98-116 (2010).

10. Modrego PJ, Ferrández J. Depression in patients with mild cognitive impairment increases the risk of developing dementia of Alzheimer type: a prospective cohort study. Arch. Neurol 61(8), 1290-1293 (2004).

11. Zhang W. Religious Participation, Gender Differences, and Cognitive Impairment among the Oldest-Old in China. J. Aging Res 2010(6), 160294 (2010).

12. Hill TD, Burdette AM, Angel JL, et al. Religious Attendance and Cognitive Functioning Among Older Mexican Americans. J. Gerontol

\section{1(1), 3-9 (2006).}

13. Inzelberg $R$, Afgin $A E$, Massarwa $M$, et al. Prayer at midlife is associated with reduced risk of cognitive decline in Arabic women. Curr. Alzheimer. Res 10(3), 340-346 (2013).

14. Kaufman $Y$, Anaki D, Binns $M$, et al. Cognitive decline in Alzheimer disease: Impact of spirituality, religiosity, and QOL. Neurol 68(18) 1509-1514 (2007).

15. Coin A, Perissinotto E, Najjar M, et al. Does religiosity protect against cognitive and behavioral decline in Alzheimer's dementia? Curr. Alzheimer. Res 7(5), 445-452 (2010).

16. Kendler KS, Gardner CO, Prescott CA. Religion, psychopathology, and substance use and abuse; a multimeasure, geneticepidemiologic study. Am J. Psychiatry154(3), 322-329 (1997).

17. Hill TD, Burdette AM, Taylor J, et al. Religious Attendance and the Mobility Trajectories of Older Mexican Americans: An Application of the Growth Mixture Model. J. Health Soc Behav 57(1), 118-134 (2016).

18. Zou J, Huang Y, Maldonado L, et al. The efficacy of religious service attendance in reducing depressive symptoms. Soc. Psychiatry. Psychiatr. Epidemiol 49(6), 911-918 (2014).

19. Fernandez A, Loukas A. Acculturation and religious coping as moderators of the association between discrimination and depressive symptoms among MexicanAmerican vocational students. J. Immigr Minor. Health 16(6), 1290-1293 (2014).

20. Balbuena L, Baetz M, Bowen R. Religious attendance, spirituality, and major depression in Canada: a 14-year follow-up study. Can J. Psychiatry 58(4), 225-232(2013).

21. Reyesortiz CA, Berges IM, Raji MA, et al. Church Attendance Mediates the Association Between Depressive Symptoms and Cognitive Functioning Among Older Mexican Americans. J. Gerontol 63(5), 480-486 (2008).

22. Foong HF, Hamid TA, Ibrahim R, et al. Moderating effect of intrinsic religiosity on the relationship between depression and cognitive function among communitydwelling older adults. Aging Ment Health 1(6), 1-6 (2017).
23. Raykov T, Marcoulides GA. A Method for Comparing Completely Standardized Solutions in Multiple Groups. Struct. Equ. Modeling 7(2), 292-308(2000).

24. Tombaugh TN, Mcintyre NJ. The mini-mental state examination: a comprehensive review. $J$. Am Geriatr. Soc 40(9), 922-935 (1992).

25. Katzman R, Zhang M, Ouang-Ya-Qu, et al. A Chinese version of the mini-mental state examination; Impact of illiteracy in a Shangha dementia survey. J. Clin Epidemiol 41(10), 971978 (1988).

26. Bravo G, Hébert R. Age- and educationspecific reference values for the Mini-Mental and modified Mini-Mental State Examinations derived from a non-demented elderly population. Int J. Geriatr Psychiatry 12(10), 1008-1018 (1997).

27. Petersen RC, Negash S. Mild cognitive impairment: An Overview. CNS Spectr 13(1), 46-53 (2008).

28. Cui GH, Yao YH, Xu RF, et al. Cognitive impairment using education-based cutoff points for CMMSE scores in elderly Chinese people of agricultural and rural Shanghai China. Acta. Neurol. Scand 124(6), 361367(2001).

29. Koenig HG, Büssing A. The Duke University Religion Index (DUREL): A Five-Item Measure for Use in Epidemiological Studies. Religions 1(1),78-85 (2010).

30. Chen $\mathrm{H}$, Wang Z, Phillips MR, et al. Internal consistency and test-retest reliability of the Chinese version of the 5-item Duke University Religion Index. Shanghai. Arch. Psychiatry. 26(5), 300-309 (2014).

31. Wang L, Wang Z, Koenig HG, et al. Interactions between Apolipoprotein E Genes and Religiosity in Relation to Mild Cognitive Impairment. Neuropsy 7(5), 640-647 (2017).

32. Marty MA, Pepin R, June A, et al. Geriatric Depression Scale. J. Am Geriatr Soc 38(12), 6370 (1990).

33. Lee YS, Park SY, Roh S, et al. The Role of Religiousness/Spirituality and Social Networks in Predicting Depressive Symptoms among Older Korean Americans. J. Cross Cult Gerontol 32(2), 239-254 (2017). 


\section{Research Zhizhong Wang}

34. Vickery, CD. Geriatric Depression Scale. Springer New York. 38 (4),709-711 (2011)

35. Chan AC. Clinical validation of the Geriatric Depression Scale (GDS): Chinese version. J. Aging Health 8(2), 238-253 (1996).

36. Briere J, Hodges M, Godbout N. Traumatic stress, affect dysregulation, and dysfunctional avoidance: a structural equation model. J. Trauma. Stress 23(6), 767-774 (2010)

37. Mackinnon DP, Fritz MS, Williams J, et al. Distribution of the product confidence limits for the indirect effect: program PRODCLIN. Behav. Res. Methods 39(3), 384389 (2007).

38. Mackinnon DP, Lockwood CM, Williams J. Confidence Limits for the Indirect Effect: Distribution of the Product and Resampling Methods. Multivariate. Behav. Res 39(1), 99128 (2004).

39. .Na KS, Jung HY, Lee SI, et al. Mediating effects of cognitive effort and depression on intelligence, memory, and executive functions in individuals with mild traumatic brain injury. Psychiatry. Investig 11(2), 112 118 (2014).

40. Paine DR, Sandage SJ. Religious Involvement and Depression: The
Mediating Effect of Relational Spirituality. J. Relig Health 56(1), 269-283 (2017).

41. Wilson RS, Bennett DA, Bienias JL, et al. Cognitive activity and incident $A D$ in a population-based sample of older persons. Neurol 59(12), 1910-1914 (2002).

42. Vail KE, Arndt J, Abdollahi A. Exploring the existential function of religion and supernatural agent beliefs among Christians, Muslims, atheists, and agnostics. Pers Soc Psychol Bull 38(10), 12881300(2012)

43. Vance DE, Webb NM, Marceaux JC, et al. Mental Stimulation, Neural Plasticity, and Aging. J. Neurosci. Nurs 40(4), 241-249 (2008).

44. Gotlib IH, Joormann J. Cognition and depression: current status and future directions. Annu Rev Clin Psychol 1(6), 285 312 (2010).

45. Withall A, Harris LM, Cumming SR. A longitudinal study of cognitive function in melancholic and non-melancholic subtypes of Major Depressive Disorder. J. Affect Disord 123(1-3), 150-157 (2010).

46. Kala M. Mehta KY, Kenneth M. et al. Whooley, and Kenneth E. Covinsky. Additive effects of cognitive function and depressive symptoms on mortality in elderly community-living adults. J. Gerontol 58(5), M462-467 (2003).

47. Krogh J, Benros ME, Jørgensen MB, et al. The association between depressive symptoms, cognitive function, and inflammation in major depression. Brain Behav. Immun 35(7), 70-76 (2014).

48. Morris JC, Cummings J. Mild cognitive impairment $(\mathrm{MCl})$ represents early-stage Alzheimer's disease. J. Alzheimers Dis 7(3), 235-239 (2005).

49. Graham JE, Rockwood K, Beattie BL, et al. Prevalence and severity of cognitive impairment with and without dementia in an elderly population. Lancet 349(9068), 1793-1796 (1997).

50. Xiaolu N, Xiaozhen L, Lin Z, et al. Prevalence of mild cognitive impairment in China冈a meta-analysis of studies in 2001-2015. Chin. J. Psychiatry 49(5), 298-306 (2016).

51. Koenig HG, Pearce MJ, Nelson B. Religious vs. conventional cognitive behavioral therapy for major depression in persons with chronic medical illness: A pilot randomized trial. The. J. of Nervous and Mental Disease 203(4), 243-251 (2015). 